\title{
Construction and its Caracteristic on the Concept of a 'Health Culture'
}

\author{
Toshiyuki TAKIZAWA \\ Depertment of public health, school of education, Ibaraki University, Mito
}

\begin{abstract}
Toward the 21st century, subjects and methods of hygiene and public health will be specialized and subdivided. However, practical approaches to human health need an integrated method focusing to a structure of human life. Under these circumstances, the concept of 'health culture'becomes prevalent. The role of hygiene related to the inprovement of life style, the development of re-cycle system, and fulfillment of a barrier-free system focussed on the handicapped and the eldery have become increasingly important.

Therefore, an increase in the recognition to the concept of 'health culture' is essential to the research of hygiene.

The purpose of this paper is to make a historical and theoritical analysis of 'health culture', in order to promote it as the leading concept of all activities concerned with health in the 21 th century. The methods of this paper are mainly historical and theoretical review.

'Health culture' was introduced in American and European societies a hundred years ago. Health culture in the USA involves knowledge and skills applied to actual daily life, refrecting pragmatism as the ideal feature of American society.

In Germany, the concept of 'Hyginishe Kultur' was established at the field of social hygiene by Grotjahn and by Fischer in the early 20th century. This concept recognized the importance of the development of culture and independence of life in labour based on the evolution of the concept of ' Hygienishe Kultur'. In Japan, under the influence of German social hygiene, the social hygienic theory flourished. A social hygienist, Tetsuo Hoshino used the term 'hygienic culture' in the context of life creation toward a healthy life.

Health culture is the total system concerning knowledge, experience, skill, and norms related to health, which has developed with the development of society. It has fundamental function promotes the creation of culture and self-cultivation of living man, whereas, it contributes to the realization of health based on individuality, in conjunction with the co-oporation of medical and health sciences. The contemporary representation of health culture includes new health care activities such as self-care, a self-help movement, and health volunteer activities. It means the basic shift of the function of health culture is from that of life style to life movement.

Nowadays, the role of hygiene in the total health care system is seriously considered. The goal, objects, and methods of hygiene should be re-evaluated under the concept of 'health culture', in order to re-define hygiene as the science for people living in a society.
\end{abstract}

Jpn J Hyg 1998; 53: 398-406.

\section{The Relationship between Stress and Health Indicators in an Urban Population - From a study of subjects selected by sex and age groups who underwent health check-ups in S city in Osaka Prefecture -}

\author{
Yuko NAKAGAWA*1, Hirono TAKABAYASHI*2, Shingo TAKAHASI*2 and Kozo TATARA*2 \\ * Department of Epidemiology and Mass examination, Osaka Medical Center for Cancer and Cardiovascular Diseases, Osaka \\ * Department of Public Health, School of Medicine, Osaka University, Suita
}

\footnotetext{
Abstract Many people in recent years are living under conditions involving various kinds of major and minor stress. This study was done to analyze how an urban population experience stress in daily life and to establish any relationship between the feeling of stress and people's daily habits or health conditions. The subjects were 2,234 residents, who availed themselves of general health check-ups in a city in Osaka Prefecture in 1992. The main results were as follows:

1) We designed and assessed a total stress score for causes of stress in daily life and a total depression score for the depressive condition of subjects.

2) The proportion of subjects who showed a high total stress score decreased with age. The proportion of subjects who showed the highest total stress score was the largest in the age group of 40-49 years for men and of 30-39 years for women.

3) The proportion of subjects who had short working hours, much leisure time available, and low total depression score and
} 
complained much about health conditions increased with age. The proportion of subjects who had the longest working hours was the largest in the age group of 30-39, that of those who had little leisure time available and showed the highest total depression score was the largest in the age group of $40-49$, and that of those who complained most about health conditions was the largest in the age group of 60-69 for men. The proportion of subjects who had the shortest sleeping hours and the longest working hours was the largest in the age group of $40-49$, that of those who had little leisure time was the largest in the age group of 30-39, and that of those who showed the highest toal depression score was the largest in the age group of 30-39 and 50-59 for women.

4) The total stress score showed significant relationships with the amount of leisure time available, the degree of complaints about health condition, and the total depression score for men and with sleeping hours, the amount of leisure time available, the degree of complaints about health condition and the total depression score for women.

5) The total stress score showed a significant correlation with high blood pressure for men and women.

6) The amount of leisure time available showed significant relationships with sleeping hours for men and working hours for men and women. The total depression score showed significant relationships with the degree of complaints of health condition for men and with sleeping and working hours, the amount of leisure time available and the degree of complaints of health condition for women.

Jpn J Hyg 1998; 53: 407-19.

\title{
Flow Cytometric Analysis of the Effects of $50 \mathrm{~Hz}$ Magnetic Fields on Mouse Spermatogenesis
}

\author{
Hiroyuki FURUYA ${ }^{* 1}$, Hiroyuki AIKAWA', , Tomoyasu HAGINO*1, Takahiko YOSHIDA*1 \\ and Kou SAKABE*2
}

${ }^{*}$ Department of Environmental Health, Division of Community and Environmental Health, Isehara

*Division of Steroid Toxicology, Department of Anatomy and Cellular Biology, Isehara

\begin{abstract}
The cellular effects of an extremely-low-frequency (ELF) magnetic field on mouse spermatogenesis were assessed by DNA flow cytometry and serum testosterone. Seven week old male ICR mice were exposed to a $50 \mathrm{~Hz}$ magnetic field the strength of which was 1.0 mTesla. Seven mice per treatment group were exposed for 13, 26, 39 or 52 days. For each experimental point, an equal number of mice per sham-treated group were used as a control and were exposed only to the background field below $1 \mu$ Tesla in the same room as the treatment group.

In the control mice, the testis cellular DNA content distribution by flow cytometory was characterized by four quantifiable populations; round spermatids (1C), spermatogonia and other diploid cells (2C), spermatogonial cells synthesizing DNA (Sphase) and primary spermatocytes (4C).

In animals exposed for 26 days the number of cells in the $4 \mathrm{C}$ and the $4 \mathrm{C}: 2 \mathrm{C}$ ratio was significantly lower, and the $1 \mathrm{C}: 4 \mathrm{C}$ ratio (meiotic transformation) was significantly higher than the corresponding control groups. In animals exposed for 52 days the cell population in $1 \mathrm{C}$ and the $1 \mathrm{C}: 2 \mathrm{C}$ ratio (total germ-cell transformation) was significantly higher, and the cell population in $2 \mathrm{C}$ was significantly lower than the corresponding control groups.

The concentration of serum testosterone in animals exposed for 13 days was significantly higher than in the corresponding control group.

These changes suggest that long-term exposure to an ELF magnetic field had a possible effect on the proliferation and differentiation of spermatogonia.
\end{abstract}

Jpn J Hyg 1998; 53: 420-5. 


\title{
The Presentation Level of Interrupted Pure Tone for Inducing the Pulse Phenomenon of Continuous White Noise
}

\author{
Kouichi MURATA*1,2, Ryouichi INABA*2 and Hirotoshi IWATA*2 \\ * Department of Audiology and Logopedics II, Japan College of Rehabilitation and Welfare Professionals, Nagoya \\ * Department of Hygiene, Gifu University School of Medicine, Gifu
}

\begin{abstract}
When two sounds which have same spectrum but different amplitude are alternated
without silent gap between the sounds,the lower amplitude sound comes to be heard as continuous. This is called "auditory induction" which is one of the most interesting auditory phenomena. The fainter sound is called inducee and the louder sound is called inducer.

The authors previously reported that the addition of interrupted pure tone to continuous white noise induced the pulsed noise. This pulse phenomenon was thought to be a kind of auditory induction;the inducer is the noise image of white noise with the silent part of interrupted pure tone and the inducee is the noise image of white noise with the pure tone part of interrupted pure tone. The authors hypothesized that an auditory filter made with the pure tone of interrupted pure tone generates the two noise images in the continuous white noise. Present experiments were made to investigate the change of "the threshold of interrupted pure tone for inducing the pulse phenomenon" (TIP) when the presentation level of continuous white noise and the frequency of interrupted pure tone are changed. TIP was constant and independent of both the amplitude of continuous white noise and the frequency of interrupted pure tone. All subjets reported that the higher the frequency of interrupted pure tone is, the harder it becomes to hear the pulsed noise image. It is supposed that this phenomenon does not contradict the theory of the auditory filter.
\end{abstract}

Jpn J Hyg 1998; 53: 426-30.

\section{Effects of Acute Endurance Exercise and 8 Week Training on the Production of Reactive Oxygen Species from Neutrophils in Untrained Men.}

\author{
Hideki SATO*1, Katsuhiko SUZUKI*1, Sigeyuki NAKAJI*1, Kazuo SUGAWARA*1, \\ Manabu TOTSUKA*2 and Koki SATO*2 \\ ${ }^{*}$ Department of Hygiene, Hirosaki University School of Medicine, Hirosaki \\ * Department of Health and Physical Education, Faculty of Education, Hirosaki University, Hirosaki
}

\begin{abstract}
We investigated the effects of acute endurance exercise and habitual physical activity for health maintenance on human neutrophil function in 12 untrained men. The acute exercise condition was a continuous exercise for 90 minutes at the intensity of $50 \%$ and $55 \%$ of maximal oxygen uptake $\left(\mathrm{VO}_{2} \max \right)$ on an ergometer. The training $r \quad$ ram was $3 \mathrm{~km}$ jogging three times per week for 8 weeks. The capacity of neutrophils to produce reactive oxygen species (ROS, , as detected with lucigenindependent chemiluminescence $(\mathrm{LgCL})$ and luminol-dependent chemiluminescence $(\mathrm{LmCL})$ on stimulation with opsonized zymosan (OZ) and phorbol myristate acetate (PMA).

As for the acute exercise effects, both $\mathrm{LgCL}$ and $\mathrm{LmCL}$ responses of neutrophils stimulated using PMA consistently increased after exercise at $50 \% \mathrm{VO}_{2} \max$, whereas those stimulated with $\mathrm{OZ}$ remained unchanged. At $55 \% \mathrm{VO}_{2} \max , \mathrm{LgCL}$ responses to both stimulants increase maximally $\mathrm{th}$ after exercise, and then decreased $3 \mathrm{~h}$ after exercise, whereas $\mathrm{LmCL}$ responses to both stimulants increased continuously after exercise at $55 \% \mathrm{VO}_{2}$ max. These phenomena observed at $55 \% \mathrm{VO}_{2}$ max compared to $50 \% \mathrm{VO}_{2} \max$ suggests the improved capacity of producing ROS neutrophils after exercise. The number of neutrophils also increased maximally $\mathrm{lh}$ after exercise, due to the mobilization of band neutrophils (shift to the left), suggesting that functional changes was associated with cell mobilization. The increase in the capacity of neutrophils to produce ROS and marked neutrophilia following the acute endurance exercise suggests that a large quantity of ROS may be produced in vivo.

As for the training effects, the $\mathrm{LgCL}$ and $\mathrm{LmCL}$ responses were maintained in the exercise group as compared to the decreased ones in the control group. The difference between the exercise group and the control group was observed only in $\mathrm{LgCL}$ response to $\mathrm{OZ}$. Humoral immune factors (IgG, IgA, IgM, C3, C4) and serum opsonic activity were also unaltered. These phenomena suggest that homeostasis might be kept constant in terms of immunity through regular physical activity.
\end{abstract}

Jpn J Hyg 1998; 53: 431-40. 


\title{
The Effects of Improved Diets on the Daily Intake of Environmental Contaminants as Calculated from Personal Food Consumption Data, by Monte Carlo Simulation
}

\author{
Hiroaki NISHIKAWA, Hiroyuki TOYOKAWA \\ Department of Public Health, Toho University School of Medicine, Tokyo
}

\begin{abstract}
In a previous paper, we discussed the Estimated Ecological Daily Intake (EEDI), which is a new method for the estimating daily intake of environmental contaminants based on individual food consumption data. This method makes it possible to identify high-risk cases, using a Monte Carlo simulation for varying contamination levels in each food item and permits epidemiological assessment of the individual, rather than the population, intake of environmental contaminants.

We attempted to identify those contaminants whose maximum dietary levels were most commonly exceeded.

The results obtained were as follows:

1) After a 1,000-fold extrapolation, performed for each person and contaminant, some cases exceeded allowable maximums in dieldrin, lead, cadmium, and total mercury. In dieldrin and lead intake, few cases exceeded dieldrin and lead maximums by a factor of 2 or 3 , but in the cases of cadmium and total mercury, individual maximum intake was significantly exceeded.

2) After estimating a high-risk individual's times of exceeding the allowance in cadmium intake with dietary improvement, we found a $10.5 \%$ excess intake after $40 \%$ improvement. Clearly, the issue of environmental contaminants exposure due to dietary intake is a significant one.
\end{abstract}

Jpn J Hyg 1998; 53: 441-6.

\section{The Development of the Japanese Version of the Maslach Burnout Inventory and the Examination of the Factor Structure}

\author{
Kazuyo HIGASHIGUCHI ${ }^{* 1,2}$, Yuko MORIKAWA*2, Katsuyuki MIURA*2, Muneko NISHIJO*2, \\ Masaji TABATA $^{* 2}$, Katsushi YOSHITA*2, Takiko SAGARA*2 and Hideaki NAKAGAWA*2 \\ *,2Department of Nursing, Kanazawa Medical University, Hospital, Ishikawa \\ * Department of Public Health, Kanazawa Medical University, Ishikawa
}

\begin{abstract}
This article presents an evaluation of the factor structures of the Japanese version of the Maslach Burnout Inventory (MBI). The MBI is a widely used psychometric instrument for measuring 'burnout' developed by Maslach and her coworkers. The MBI consists of four subscales: Emotional Exhaustion, Personal Accomplishment, Depersonalization, and Involvement. The MBI was translated into Japanese along with a back-translation and was administered to a sample of 267 nurses.

Various psychometric analyses showed that the Japanese version of the MBI has high reliability for the 22 items scored for the frequency dimension. The factor analysis using principal factoring with an oblique rotation resulted in three factor structures that had different implications from the MBI: Emotional Exhaustion/Depersonalization, Personal Accomplishment, and Physical Exhaustion. The correlationship between the MBI and the General Health Questionnaire (GHQ), measures of depression, showed that burnout was a unique phenomenon.
\end{abstract}

Jpn J Hyg 1998; 53: 447-55. 


\title{
NK Cell Activity and Subsets of Truck Drivers along with Related Factors
}

\author{
Ming XU*1, Yasushi MIURA*1, Fumiko NAGAO*2, \\ Takashi MUTO*1 and Ko OKUMURA*2 \\ * Department of Public Health, Juntendo University, School of Medicine, Tokyo \\ *2 Department of Immunology, Juntendo University, School of Medicine, Tokyo
}

\begin{abstract}
We investigated the immunity and the main factors that affect the immune system of 19 truck drivers as the experimental group and 27 office workers as the control group at a transit corporation in Tokyo; all subjects in both groups were examined through an assay of NK activity and NK subsets before and after work. At the same time, they were asked to complete a questionnaire on their working hours per day (WHPD) and driving hours per day (DHPD), in addition to the Health Practice Index (HPI), Self-Rating Depression Scale (SDS), Stress Tolerance Check List (STCL), and Symptom Checklist (SCL). The results obtained were as follows:

1. Before work, no difference was seen in NK activity between the experimental group and the control group, with both groups showing E:T ratios 5:1 to 20:1. After work, the NK activity ( $E: T=20: 1)$ of the experimental group was significantly lower than that of the control group.

2. In the experimental group, the NK activity and NK subsets $\left(\mathrm{CD} 3{ }^{-} \mathrm{CD} 16^{\circ} \mathrm{CD} 56^{*}, \mathrm{CD} 3{ }^{-\mathrm{CD}} 6^{+}, \mathrm{CD} 3 \mathrm{CD} 16^{*}\right)$ of the peripheral blood lymphocytes after work were significantly lower than before work $(p<0.05, p<0.01, p<0.01)$. After more than five driving hours, the value of the $\mathrm{CD} 3 \mathrm{CD} 16^{\circ} \mathrm{CD} 56^{+}$subset was significantly lower than that after fewer driving hours.

3. Correlation coefficients were calculated based on the average value of $\mathrm{NK}$ activity $(\mathrm{E}: \mathrm{T}=20: 1), \mathrm{CD} 3{ }^{\circ} \mathrm{CD} 16{ }^{\circ} \mathrm{CD} 56^{\circ}$ subset, and WHPD, DHPD, and HPI in the driving group. NK activity ( $E: T=20: 1)$ and DHPD were found to be negatively correlated $(\mathrm{r}=-0.28)$, as were the CD3CD16 CD56' subset and WHPD ( $r=-0.43)$ and the CD3CD16 CD56 subset and DHPD ( $r=-$ 0.63). On the other hand, NK activity ( $E: T=20: 1)$, the $C D 3 \cdot C D 16 \cdot C D 56$ subset and HPI were found to be positively correlated ( $r=0.41$ and 0.33 ).
\end{abstract}

Jpn J Hyg 1998; 53: 456-62.

\section{The Effects of Fluid Ingestion and its Composition on Uric Acid Metabolism during High Intensity Long Term Exercise.}

\author{
Toshimasa TAKANISHI*1,4, Naoto KIMURA*2, Takashi ITO*2, \\ Yoshio MOROTOMI*3 and Toru ITANI*4 \\ * College of Integrated Arts and Sciences, Osaka Prefecture University, Sakai \\ * Department of Public Health and Preventative Medicine, Nippon Sport Science University, Tokyo \\ *s Department of Health Education, Nippon Sport Science University, Tokyo \\ * Department of Hygiene and Occuaptional Health, Nagoya City University Medical School, Nagoya
}

\begin{abstract}
The purpose of this study was to analyze the effects of fluid ingestion and its composition on uric acid metabolism after exercise. Six healthy males volunteered for the study which was comprised of three different experiments; Exp.1, Exp.2, and Exp.3. In all the experiments, subjects performed treadmill exercise $\left(70 \% \mathrm{VO}_{2} \max \right)$ for 70 minutes respectively. For seven hours after exercise, subjects ingested mineral water at $10^{\circ} \mathrm{C}$ ad-lib in Exp.1, 1.5 times the volume of mineral water consumed in the first experiment in Exp.2, and the same volume of sports drink as in the first experiment in Exp.3. No significant differences were observed in oxygen uptake and heart rate during exercise among the three experiments, so it was considered that the produced serum uric acid (SUA) levels in the three experiments were about the same level. However, the decrease in SUA, urinary uric acid excretion (UUA), clearance of uric acid (CUA) and fractional excretion of uric acid (FEUA) in Exp.3, in which the sports drink was consumed instead of mineral water were higher than in Exp.1 and Exp.2. On the other hand, no significant differences were found in Exp.2 and Exp.3. A significant relationship between UUA and FEUA was found among the three experiments, while there was no correlation between UUA and urine volume.

These results show that; 1) the sports drink ingestion can increase the efficiency of recovery from high serum uric acid after exercise, 2) the increase in uric volume due to high mineral water intake does not elevate UUA, and 3) the increase in UUA due to sports drink ingestion was associated with the increase of FEUA.
\end{abstract}

Jpn J Hyg 1998; 53: 463-9. 


\title{
An Analysis on a Relationship between Perinatal Mortality and Live Births of Low Birthweight, in Kumamoto Prefecture, 1968-1994
}

\author{
Kimiyo UEDA*1, Mitsukazu ONOMICHI*1, \\ Koichi HARADA*2, Takashi MIYAKITA*2 and Atsushi UEDA*2 \\ ${ }^{*}$ Kumamoto University College of Medical Science, Kumamoto \\ * Department of Hygiene, Kumamoto university School of Medicine, Kumamoto
}

\begin{abstract}
The aims of this investigation were to describe the relationship between perinatal mortality rates and the proportions of live births among low birth weight (LBW) infants from 1968 to 1994, and to determine risk factors in infants of low birth weight. Using vital statistics from 1968 to 1994 of the Japanese government and the Kumamoto Prefecture and Maternal and Child Health Statistics of Japan from 1968 to 1995, perinatal deaths and live births of infants of low birth weight were studied according to national statistics criteria.

In Japanese and Kumamoto Prefecture, there was an association between perinatal deaths and live births of LBW. The significant decline of perinatal mortality rates from 1968 to 1976 , of which the fetal death ratio at 28 weeks and over mostly declined, was closely related to the decline of live births of LBW. In this period, the improvement of socioeconomic conditions and the comprehensive health care provided by the government contributed in improving perinatal mortality rates. From 1977 to 1988 , the annual variation of Kumamoto was different from that of all Japan. Both perinatal mortality rates continued to decline due to a general decrease in early neonatal mortality rates. The number of Live births of LBW infants in Kumamoto prefecture increased in 1977 and once again started to decline in 1982. In 1977, the insufficient maternal-child health care and the increase of female workers contributed to increasing rates of live births of LBW. Advances in neonatal medicine contributed to the increase in survival rates of infants of LBW. Although, after 1982, the improvement of maternal- child health care and the perinatal care system contributed to the declining rates of live births of LBW. On the other hand, those rates in all Japan continued to gradually increase from 1977 to 1988 . After 1989 , perinatal mortality rates continued to decline, and live births of LBW continued to increase in both Kumamoto prefecture and all Japan. These results were contrary to the above results from 1968 to 1976 . It was considered that medical advances in the care of pregnant women and neonates increased survival rates of the LBW.

In future, the perinatal mortality rates will appear to approach a minimum constant, that is, a minimum of fetal death rates. It is important to reduce the number of LBW infants, particularly birthweight of $2000 \mathrm{~g}-2499 \mathrm{~g}$, with maternal- child health care.
\end{abstract}

Jpn J Hyg 1998; 53: 470-6.

\section{Study of Skin Temperature, Microclimate and Confort of Clothing of Old Females at Rest Ambient Temperature $\cdot$ Humidity : $30^{\circ} \mathrm{C} \cdot$ R.H.80\%, 30 $\mathrm{C} \cdot$ R.H. $45 \%, 20^{\circ} \mathrm{C} \cdot$ R.H.45\%}

\author{
Yoshiko NAKAZATO*1, Yayoi FURUMATSU*2 and Kenichi NAKAMURA*3 \\ *University of Tokyo Kasei, Tokyo \\ * Jumonji Women's College, Saitama \\ * Department of Hygiene and Preventive Medicine, Showa University School of Medicine, Tokyo
}

\footnotetext{
Abstract Old females are compared to young females for the purpose of studying the difference in comfort caused by the environmental variables of temperature and humidity as well as the form of clothing.

Eight experiments were performed in three settings : (a) $30^{\circ} \mathrm{C}$ R.H.80\%; $30^{\circ} \mathrm{C}$ R.H.45\%; and $20^{\circ} \mathrm{C}$ R.H.45\%. The ages of the subjects range from 62 to 68 (Mean=65.17, S.D. $=1.68$ ) among old females and from 20 to 23 (Mean=20.83, S.D. $=0.76$ ) among young females. The following results were obtained:

(1) The young females were sensitive to hot temperatures, while the old females were not. On the other hand, the old females were more sensitive to cold temperatures, under $20^{\circ} \mathrm{C}$ R.H. $45 \%$, than the young females. In temperatures under $30^{\circ} \mathrm{C}$ R.H.80\%, the heat radiation from the young females was higher than that of the old females. Under $20^{\circ} \mathrm{C}$ R.H. $45 \%$, the heat radiation from the old females was higher than from the young females. The old females are thought to decline in physiogenic function due to enduring both hot and cold temperatures.

(2) The correlation between the temperature in clothes and comfort among the old females is not different from the same correlation among the young females. This conclusion agrees with previously published studies of the young females.
} 
(3) Skin temperature and bloodstream are measured, according to clothing form. As a result, a long skirt is the highest in thermal insulation, long pants the next highest, and a short skirt is the lowest.

(4) The effect of thermal insulation provided by a lap robe was tested in both groups. The lap robe was found to be more effective for the older group than the younger in temperatures under $20^{\circ} \mathrm{C}$ R.H.45\%. Hence, the role of clothes in offsetting for the decline in the thermoregulation function that compensates for environmental change is more important for old females than for young.

Jpn J Hyg 1998; 53: 477-88. 